\title{
Nephrectomy and Tumor Thrombectomy with Concurrent Coronary Artery Bypass Graft for an HMB-45-Negative Giant Classical Renal Angiomyolipoma with Venous Extension
}

\author{
(1) Kathleen Lockhart'1, (1) Nathan Shugg1', (1) Taranpreet Singh2, (1) Albert Tiu1 \\ ${ }^{1}$ John Hunter Hospital, Department of Urology, New Lambton Heights, New South Wales, Australia \\ 2John Hunter Hospital, Department of Cardiothoracic Surgery, New Lambton Heights, New South Wales, Australia
}

\begin{abstract}
A 72-year-old woman was found to have a giant right renal mass $(11.5 \mathrm{~cm})$ suggestive of an angiomyolipoma (AML) on computed tomography imaging with atypical heterogeneity and grade III tumor thrombus extension into the inferior vena cava (IVC). Significant ischemic heart disease prompted a right radical nephrectomy on cardiopulmonary bypass with tumor thrombectomy and concurrent coronary artery bypass grafts. The patient recovered well and her histopathology confirmed a benign classical (non-epithelioid) renal AML, which was HMB-45 negative. AMLs are benign mesenchymal renal neoplasms, but surgical excision is indicated if malignant characteristics are present on imaging. HMB-45-negative AMLs are exceedingly rare, and to our knowledge, this is the first nephrectomy for an AML of this size and immunohistochemistry profile with IVC extension.
\end{abstract}

Keywords: Renal tumor, nephrectomy, angiomyolipoma

\section{Introduction}

Angiomyolipomas (AMLs) are the most common benign mesenchymal renal neoplasm, which are comprised of typical "triphasic" mature adipose tissue, smooth muscle, and dysmorphic blood vessels (1). Renal AMLs occur in 0.2-3.0\% of the population, represent $1-2 \%$ of all resected renal masses, and have a female predominance (estimated 4:1) $(1,2)$. They are associated with tuberous sclerosis complex (TSC), and rarely with lymphangioleiomyomatosis (LAM), although most occur sporadically (1). "Giant" AMLs are defined as $>10 \mathrm{~cm}$ in size. Epithelioid variants (8\% of all renal AMLs) are listed separately in the World Health Organization classification of renal tumors as they are characterized by aggressive behavior and are the only AMLs with malignant potential (2). For classical AMLs to have significant venous extension and malignant-appearing characteristics on imaging is extremely rare.

\section{Case Report}

A 72-year-old female patient was found to have incidental findings of cystic lung disease, and a large right renal mass (maximum diameter of $11.5 \mathrm{~cm}$ ) on staging computed tomography (CT) during the workup of breast cancer, for which she had a left radical mastectomy. Her only other background was ischemic heart disease and hypertension. No prior imaging was available for comparison.

The CT appearance was consistent with an $A M L$, and hypodensity indicated high-fat content. However, the mass demonstrated several atypical features, including heterogeneity with prominent central vessels from the right renal artery, grade III tumor thrombus extension into the inferior vena cava (IVC) causing $>50 \%$ occlusion, and displacement of the right ureter, though without obstructive features (Figure 1). Thus, concern for malignancy prompted consideration of nephrectomy.

Correspondence: Kathleen Lockhart MD, John Hunter Hospital, Clinic of Urology, New Lambton Heights, New South Wales, Australia E-mail: klockhart19@gmail.com ORCID-ID: orcid.org/0000-0003-1426-9599

Received: 16.11 .2020 Accepted: 23.09.2021

Cite this article as: Lockhart K, Shugg N, Singh T, Tiu A. Nephrectomy and Tumor Thrombectomy with Concurrent Coronary Artery Bypass Graft for an HMB45-Negative Giant Classical Renal Angiomyolipoma with Venous Extension. J Urol Surg 2021;8(4):288-290.

๑Copyright 2021 by the Association of Urological Surgery / Journal of Urological Surgery published by Galenos Publishing House. 
The routine preoperative investigation included spirometry, carotid Doppler ultrasound, echocardiogram, and a CT, which was followed by a percutaneous coronary angiogram that confirm significant coronary artery disease. Following the multidisciplinary discussion with the urology and cardiothoracic teams, the patient proceeded to an open right radical
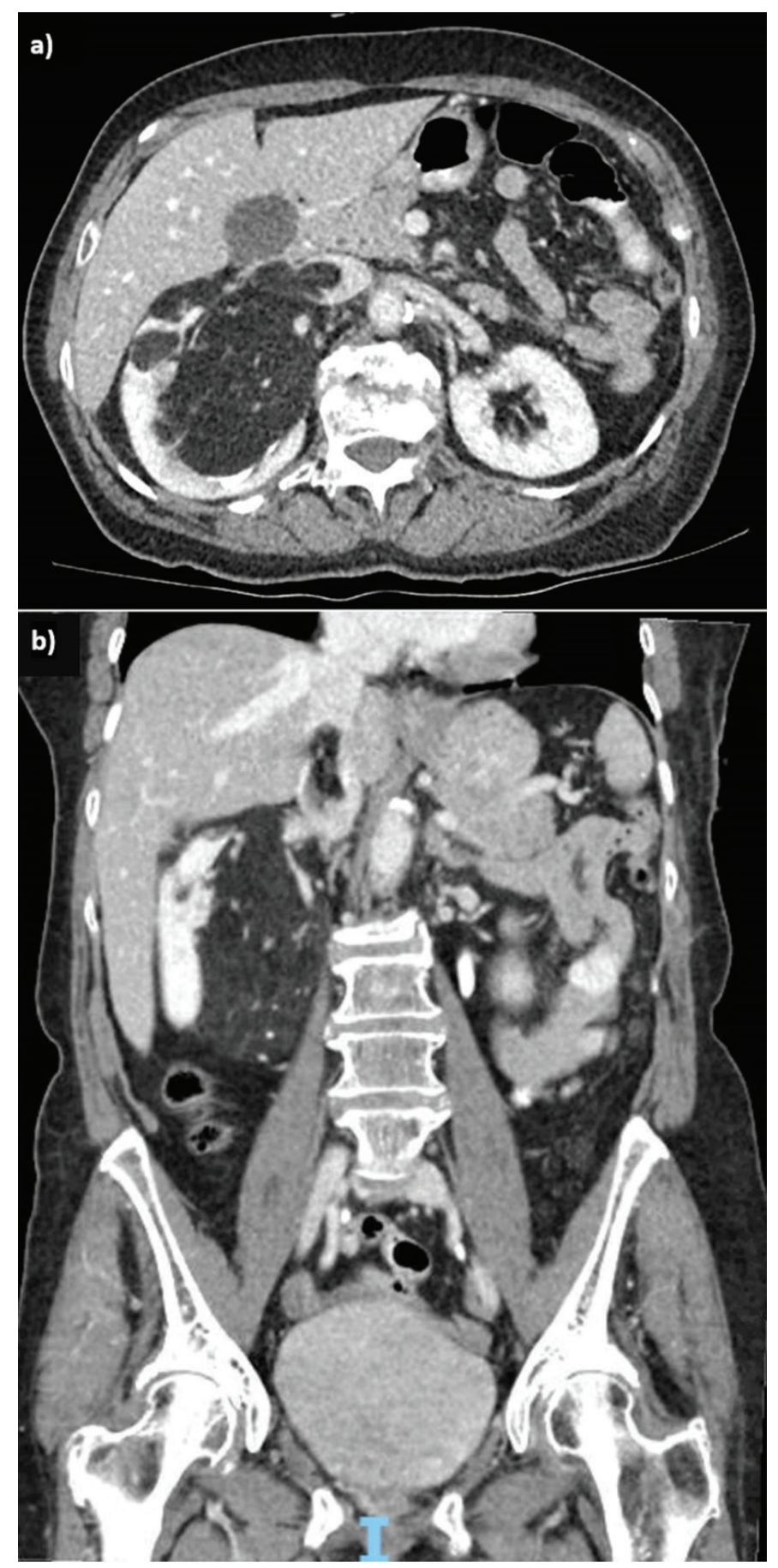

Figure 1. a) CT venous phase axial view demonstrating right renal tumor with venous extension, b) CT venous phase coronal view

CT: Computed tomography nephrectomy with IVC thrombectomy and coronary artery bypass graft (CABG).

A midline laparotomy was performed and the second part of the duodenum was kocherized, demonstrating an $11 \mathrm{~cm}$ right renal mass. The right renal artery was isolated in the inter-aortocaval region and ligated between vicryl ties. The liver was mobilized before the incision was extended to include sternotomy. The right saphenous vein was harvested and then the patient was placed on cardiopulmonary bypass, with deep hypothermic arrest, and CABG was completed by the cardiothoracic team (saphenous vein graft-obtuse marginal artery-right posterior descending artery). The aorta was clamped, the perfusion pump stopped, and the IVC was opened. The tumor thrombus was extended beyond the level of the hepatic veins (level III according to the Mayo staging system) but was not adherent and was milked back and removed; no reconstruction was required. The nephrectomy was then completed and the IVC was closed with 5-0 prolene. A drain was placed in the renal bed before closing in layers.

The total cardiopulmonary bypass time was $1 \mathrm{~h}$ and $39 \mathrm{~min}$, with a cross-clamp time of $40 \mathrm{~min}$ and deep hypothermic arrest for $16 \mathrm{~min}$ at a temperature of $25^{\circ} \mathrm{C}$.

The patient was extubated on postoperative day 1 and recuperated well in the intensive care unit with only brief transaminitis and mild acute renal impairment, which improve as evidenced by a creatinine of $94 \mu \mathrm{mol} / \mathrm{L}$ and glomerular filtration rate of $52 \mathrm{~mL} / \mathrm{min} / 1.73 \mathrm{~m}^{2}$ at discharge. She was discharged home after an uneventful inpatient recovery including a period of rehabilitation.

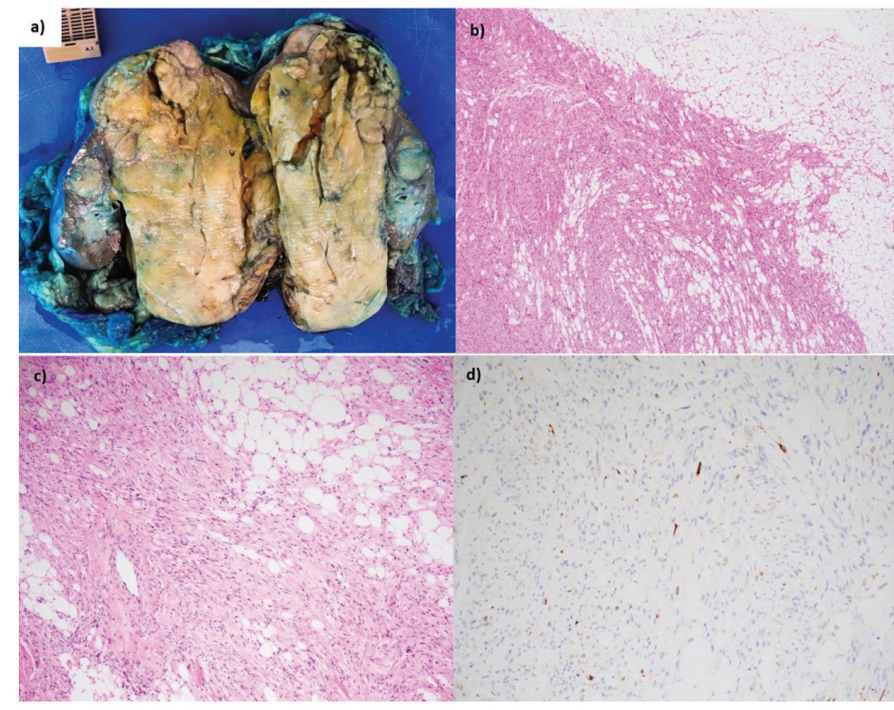

Figure 2. a) Macroscopic view of divided nephrectomy specimen exhibiting central pale yellow tumor, b) 40x magnified view of the lipomatous and myoid tumor (hematoxylin \& eosin staining), c) 100x magnified view of the smooth muscle, fat, and vessel components of the tumor, d) HMB-45 staining 
Pathological examination of the excised kidney demonstrated no extension into the perinephric fat (no lymph nodes were included). The tumor did not infiltrate the identified adrenal gland but invaded the renal sinus and the IVC. The tumor was composed primarily of mature adipose cells, as well as smooth muscle and blood vessels. No epithelioid component was identified, thus confirming a classical AML (Figure 2). The immunoprofile of the tumor was predominantly negative for HMB-45 and desmin, with positive focal cytoplasmic melan-A.

Cystic lung lesions that were seen on initial CT suggest the possibility of associated LAM in TSC. LAM is more typically diagnosed by the fourth decade and is characterized by desmin and HMB-45 positivity. Perioperative spirometry was superior to age-adjusted values, therefore no intervention had been deemed necessary preoperatively, and the patient was referred to the Respiratory team for further investigation.

\section{Discussion}

Giant AMLs with venous extension past the level of the hepatic veins pose a unique management dilemma. HMB-45 negative AMLs are exceedingly rare, and to our knowledge, this is the first nephrectomy for an AML of this size with IVC extension and immunohistochemistry profile.

Imaging is often adequate for diagnosis. AMLs tend to be highly echogenic on ultrasound, and the presence of macroscopic fat with a typical appearance on CT is considered diagnostic (although not pathognomonic). Magnetic resonance imaging, biopsy, or excision may be required for the diagnosis of the 5\% of AMLs that are fat-poor, as carcinoma cannot otherwise be excluded (1-3). AMLs are the most common renal neoplasm associated with spontaneous perirenal hemorrhage, followed by renal cell carcinomas. Venous extension of AMLs is extremely atypical, but risk factors include right-sided tumors and large size $(2,4)$. In very rare cases, intra-cardiac extension has also been reported (at least six case reports in the literature). Large AMLs with venous extension may include complications such as hypertension and its secondary complications and pulmonary fat embolism in some cases, which prompt the insertion of a preoperative IVC filter $(2,4)$.

Several treatment options are available; if AMLs meet the criteria for intervention, a minimally invasive and nephronsparing approach is preferred where feasible. Options include angioembolisation, partial (including laparoscopic or robotic approach) nephrectomy, and radiofrequency/cryo/microwave ablation $(3,5)$. However, radical surgical intervention is indicated if malignancy is suspected (5). Complete excision may also be preferred in the presence of symptomatic hemorrhage, risk of aneurysmal rupture, and factors precluding minimally invasive options (5).

Histopathology in this case notably confirmed a classical rather than epithelioid AML, although interestingly with a rare HMB45 negative immunoprofile. Epithelioid variants (8\% of all AMLs) tend to be associated with TSC and are characterized by aggressive and malignant behavior (1).

Therefore, renal AMLs may necessitate excision if the risk of malignancy or size-related complications is suspected; in this case, a classical AML (HMB-45 negative) was found despite its invasive characteristics. A significant proportion of AMLs are associated with TSC, and this should always be considered. Thorough cardiopulmonary investigation in preoperative planning is vital to optimizing overall outcomes; in this case, precipitating a concurrent $\mathrm{CABG}$ procedure.

\section{Ethics}

Informed Consent: Informed consent was obtained.

Peer-review: Externally and internally peer-reviewed.

\section{Authorship Contributions}

Concept: K.L., T.S., A.T., Design: K.L., Data Collection or Processing: K.L., N.S., Analysis or Interpretation: K.L., N.S., T.S., A.T., Literature Search: K.L., N.S., Writing: K.L., N.S.

Conflict of Interest: No conflict of interest was declared by the authors.

Financial Disclosure: The authors declared that this study received no financial support.

\section{References}

1. Jinzaki M, Silverman SG, Akita H, Nagashima Y, Mikami S, Oya M. Renal angiomyolipoma: a radiological classification and update on recent developments in diagnosis and management. Abdom Imaging 2014;39:588604.

2. Bakshi SS, Vishal K, Kalia V, Gill JS. Aggressive renal angiomyolipoma extending into the renal vein and inferior vena cava - an uncommon entity. Br J Radiol 2011;84:e166-e168.

3. Flum AS, Hamoui N, Said MA, Yang XJ, Casalino DD, McGuire BB, Perry KT, Nadler RB. Update on the Diagnosis and Management of Renal Angiomyolipoma. J Urol 2016;195:834-846.

4. Fujiwara M, Kawamura N, Okuno T. Preoperative inferior vena cava filter implantation to prevent pulmonary fat embolism in a patient showing renal angiomyolipoma extension into the renal vein: A case report and literature review. J Rural Med 2018;13:181-184.

5. Sivalingam S, Nakada SY. Contemporary minimally invasive treatment options for renal angiomyolipomas. Curr Urol Rep 2013;14:147-153. 